\title{
Disparity increase with convergence for constant perceptual criteria
}

If a depth interval is matched to an egocentric distance, the disparity required increases with convergence by 50 to $130 \%$ (depth micropsia). If a depth interval is matched to a frontal extent of constant visual angle, disparity again increases with convergence, but the proportion increase is slightly smaller. This difference is attributed to the previously established effect of convergence on perceived size (size micropsia).

Gogel (1958) had observers set a stereoscopically presented disk behind a frontal extent of constant visual angle so that the depth interval between the disk and the extent and the width of the frontal extent were perceptually equal. As distance to the extent decreased (1.e., as convergence increased), the group mean disparity between the disk and the frontal extent increased. Foley (1967) had observers match the distance between two points separated in depth with the distance from themselves to the nearer point. Here the range of convergence was small and the effect small, but again disparity increased with convergence. The present study was designed to measure this increase for individual observers in the two tasks.

The apparatus used was a polarization stereoscope (Foley, 1964). This instrument presents a three-dimensional configuration of small, point-like light sources to the observer (O). Each point is simulated by a palr of horizontally separated bulbs, one member of which is visible to each eye as shown in Fig. 1. The stereoscopic presentation is achieved by means of properly oriented pleces of Polaroid fllm, one in front of each bulb and one in front of each eye. The bulbs are in a frontal plane at a distance of $2 \mathrm{~m}$. Points behind this plane are simulated by using uncrossed Polaroids, as shown; points in front of the plane are simulated by crossing the Polaroids. The simulated distance of a point is varied by changing the horizontal separation of the two lights. Thls separation can be varied continuously by $O$ or E. Each has a pair of buttons which control a reversible motor (60 rpm); the motor drives a screw (40 threads/in.) that moves the lights together or apart. The lights are about 2 log units above foveal threshold and are matched for brightness. The field is completely dark except for the lights. The $O^{\prime} s$ head is held fixed by means of a bite. Results reported here were obtained with free eye movement, but the effect has since been replicated with fixation on one point.
The same three Os were used in both experiments. Two had acuity of better than $1 \mathrm{~min}$ and stereoacuity better than $30 \mathrm{sec}$, uncorrected. The third wore contact lenses and met these same standards with the correction. All were experienced in this type of experiment.

The depth-distance matches were made first. Two simulated lights were presented, one in the median plane, F, and the other $6^{\circ}$ to the right of it, N. The light on the right was fixed during every trial. The task was to adjust the distance to $F$ so that $F$ was behind $\mathrm{N}$ and the distance $\mathrm{FN}$ equaled the distance ON from the observer to $N$ (see insert, Fig. 2). The independent variable was the simulated distance to $\mathrm{N}$. This was measured in terms of the convergence angle $\left(\gamma_{n}\right.$ in Fig. 1) at $N$. The horizontal disparity, $\Gamma$, is the difference between the two visual angles, $\alpha_{1}-\alpha_{r}$, which equals the difference between the two convergence angles, $\gamma_{n}-\gamma_{f}$. Values of $\gamma_{n}$ ranged from 20 to $250 \mathrm{~min}$. This corresponds to a range of distance from less than one to several hundred meters. The exact values differ from $O$ to $O$ due to differences in interpupillary distance and

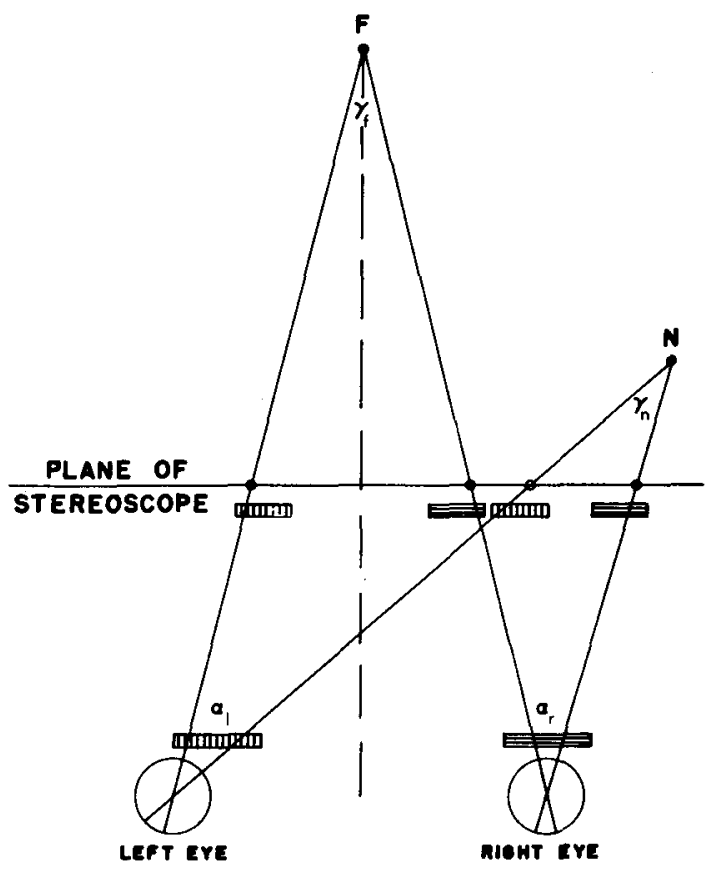

Fig. 1. Schematic illustration of the apparatus. Open circles represent point sources of light; closed circles, simulated points; $\gamma_{\mathbf{n}}, \gamma_{\mathbf{f}}$, convergence angles; $\alpha_{\mathbf{l}}, a_{\mathbf{r}}$, visual angles. 


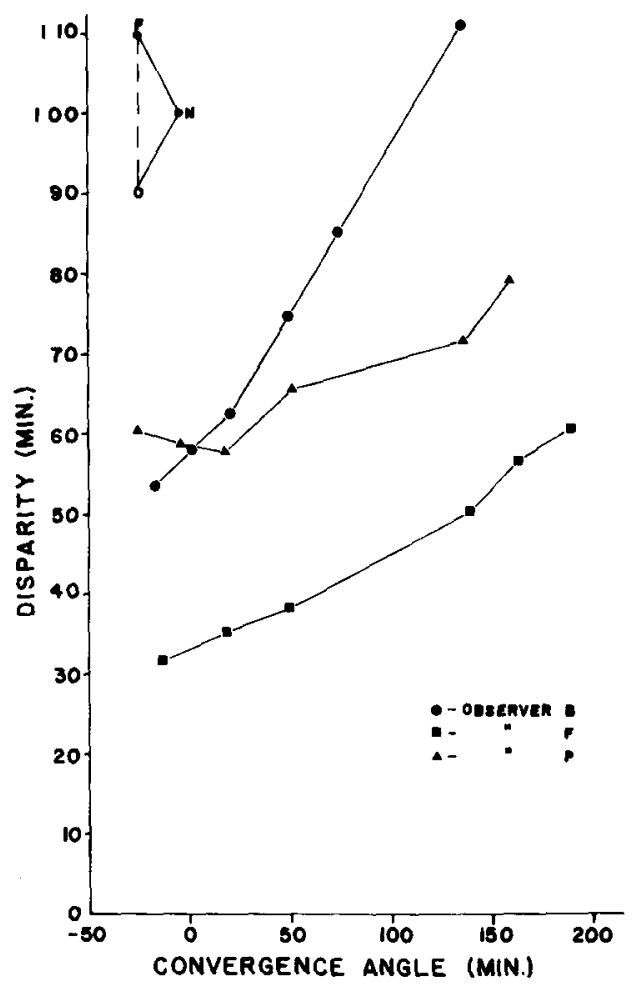

Fig. 2. Disparity for depth-distance match (FN $=$ NO) as a function of the convergence angle at the far point. $N=42$.

variations necessary to keep all the settings within the range of the apparatus while covering as large a range as possible. The results are given in Fig. 2. Each point represents the mean of 42 settings. Disparity is plotted against $\gamma_{f}$ rather than $\gamma_{n}$ since an earlier study (Foley, 1967) indicated that the function passes through a minimum at $\gamma_{f}=0$. This effect is evident here for observer $P$, who is the only one with two values of $\gamma_{f}<0$. The standard deviation of the settings increased with convergence. Values ranged from 3.3 to $12.4 \mathrm{~min}$.

For the depth-size matches the lights were arranged in exactly the same way as described above. Here the task was to set $F$ as far behind the frontoparallel plane containing $\mathrm{N}$ as $\mathrm{N}$ was to the right of the direction of $F$ (see insert, Fig. 3). The procedure was the same as in the depth-distance matches. The results are given in Fig. 3. Each point represents the mean of 45 settings. Standard deviations increased with convergence. Values ranged from 2.5 to $8.5 \mathrm{~min}$.

It is clear that disparity increases significantly with convergence angle in both tasks. The relation may be reasonably well represented by a power function of the form:

$$
\Gamma=A Y_{f}^{B}+\Gamma_{0}
$$

where $\Gamma$ is disparity; $\gamma_{f}$, convergence angle; and $A_{\text {, }}$ $B$, and $\Gamma_{0}$ constants which depend both on the $O$ and on the task, $\Gamma_{0}$ being the intercept of the curve with the $\Gamma$-axis. Inspection of Figs. 2 and 3 suggested that the change might be a proportional one, the proportion increase in disparity being the same function of convergence angle in both tasks. If this is true and if equation (1) describes the results, the proportion increase in disparity is given by:

$$
\frac{\Gamma-\Gamma_{o}}{\Gamma_{0}}=C \gamma_{f}^{B},
$$

where $\mathrm{C}=\mathrm{A} / \Gamma_{0}$, and $\mathrm{C}$ and $\mathrm{B}$ are constants, independent of the task.

To test this notion $\Gamma_{0}$ was estimated for each of the six sets of data and the proportion increase (left side of equation (2)) calculated for each point. Estimates of $\Gamma_{0}$ were made by plotting the relation $\Gamma-\Gamma_{0}$ versus $\gamma_{f}$ on $\log -\log$ coordinates and adjusting the value of $\Gamma_{0}$ until linearity was obtained. Proportion increase is plotted against convergence angle in Fig. 4. Only points for which convergence angle is positive are included. For all Os, points for the depth-size task lie slightly below those for the depth-distance task.

The results imply that, if one were to present a two-point configuration of constant disparity at various distances, the depth/distance and depth/size ratios would decrease as convergence increased. This is reminiscent of the fact that frontal extents of constant visual angle appear to decrease in size or recede (i.e., size/distance ratio decreases) as convergence increases (Heinemann et al, 1959; McCready, 1965).1 This phenomenon has traditionally been called accommodation-convergence micropsia. Here it will be called size micropsia to distinguish it from depth micropsia.

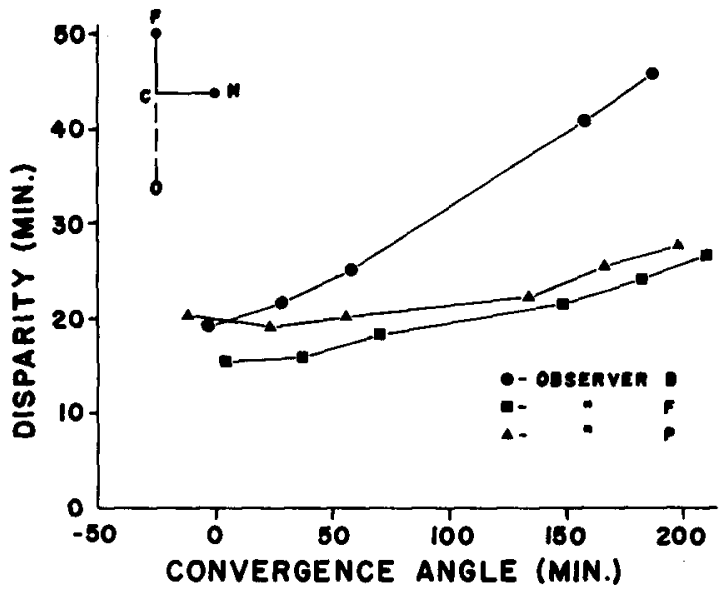

Fig. 3. Disparity for size-distance match $(\mathrm{FC}=\mathrm{CN})$ as a function of the convergence angle at the far point. $N=45$. 


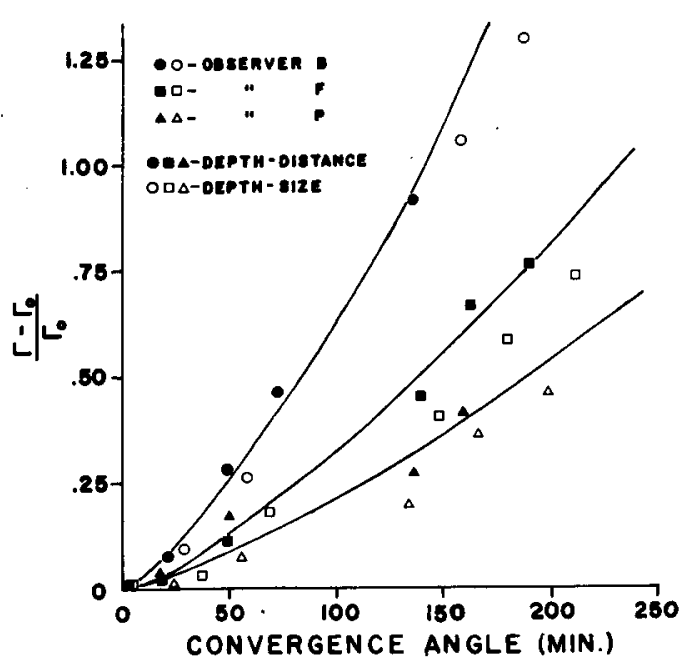

Fig. 4. Proportion increase in disparity in the two tasks as a function of the convergence angle at the far point.

Foley (1967), having found depth micropsia in the depth-distance task, hypothesized that it and size micropsia might be quite closely related effects of the same basic process. In both cases increasing convergence appears to have the same effect as would a demagnification of the retinal images. As convergence increases, visual angle must increase to maintain a constant size/distance ratio. Likewise disparity must increase to maintain a constant depth/ distance ratio. Since disparity is simply the difference between the visual angles in the two eyes, a demagnification-like process might decrease both by the same proportion and therefore require both to be increased by the same proportion to maintain constant effects. This hypothesis, however, is not consistent with the measured magnitudes of the two phenomena. In measuring size micropsia, Heinemann et al (1959) found only a 5 to $10 \%$ increase in visual angle over the range in which a 50 to $130 \%$ increase in disparity was found here. Size micropsia appears to be of lesser magnitude than depth micropsia.

The results of the depth-size matches are consistent with this interpretation. To the extent that size micropsia is present, proportion increase in disparity (Fig. 4) would be expected to be less in the depth-size task than in the depth-distance task, as was found. That the depth-size increase is only slightly less suggests that the size micropsia effect is small. 2

If the depth-size data represent the combined effect of the two types of micropsia, then only the depth-distance data can be taken as a measure of depth micropsia. 3 Accordingly equation (2) was fitted to the depth-distance data by applying the least squares criterion to the relation between the logarithms of the variables. Since two free parameters yield very little advantage over one in the goodness of fit achieved, the mean exponent yielded by this procedure, 1.33, was assumed to hold for all three sets of data. The data were then fitted by the function $\mathrm{C}_{\mathrm{f}}{ }^{1.33}$, applying least squares directly. These fitted curves are shown in Fig. 4. The coefficient $\mathrm{C}$ provides a measure of depth micropsia. Values are: Observer B, .00137; Observer F, .00071; Observer P, .00046.

Since, as convergence increases, more disparity is required to produce a given perceptual effect, then one might expect that stereoacuity would decrease as convergence increases. The data on this question are contradictory. In studies where secondary depth cues were excluded, Ogle (1958) and Jameson and Hurvich (1959) found stereoacuity to be independent of distance. Amigo (1963) found increasing stereoacuity up to $1 \mathrm{~m}$ and a decrease between 1 and $2 \mathrm{~m}$. More recently Brown, Ogle, and Reiher (1965) found an increase in the range of .4 to $1 \mathrm{~m}$ for two out of three subjects, while a third had constant stereoacuity from .4 to $6 \mathrm{~m}$. Although these studies differ from the present one in several respects, the failure to find increasing stereoacuity at distances greater than a meter is puzzling in the light of the present results. If depth micropsia is a demagnification-like process, precision should depend on convergence in the same way that central tendency does.

Depth micropsia contributes toward the veridicality of space perception. As a depth interval of constant physical extent moves toward an observer, disparity increases approximately inversely with the square of the distance. Due to depth micropsia the effectiveness of disparity falls off as distance increases, but at a lower rate. The net effect is that perceptual relations between depth, size, and distance are closer to veridicality than if micropsia were absent. In spite of micropsia, however, in stereoscopic vision perceived spatial relations remain markedly nonveridical.

\section{References}

Amigo, G. Variation in stereoscopic acuity with observation distance, J. Opt. Soc. Amer., 1963, 53, 630-635.

Brown, J. P., Ogle, K. N., \& Reiher, L. Stereoscopic acuity and observation distance. Invest. Ophthal., 1965, 4, 894-900.

Foley, J. M. Desarguesian property in visual space. J. Opt. Soc. Amer., 1964, 54, 684-692.

Foley, J. M. Binocular disparity and perceived relative distance: An examination of two hypotheses. Vis. Res., 1967, 7, 655-670.

Gogel, W. C. The effect of convergence and angular size upon the computed value of a hypothetical observer constant in binocular vision. U. S. Army Medical Research Laboratory, Fort Knox, Report No. 372, 1958. 
Heinemann, E. G., Tulving, E., \& Nachmias, J. The effect of oculomotor adjustments on apparent size, Amer. J. Psychol., 1959, $72,32-45$.

Jameson, D., \& Hurvich, L. M. Note on factors influencing the relation between stereoscopic acuity and observation distance. J. Opt. Soc. Amer., 1959, 49, 639.

McCready, D. W., Jr. Size-distance perception and accommodationconvergence micropsia - a critique. Vis. Res.. 1965, 5, 189-206.

Ogle, K. N. Note on stereoscopic acuity and observation distance. J. Opt. Soc. Amer., 1958, 48, 794-798.

\section{Notes}

1. A decrease in size/distance ratio might be a better characteriza of accommodation-convergence micropsia than the classical one. An extent does not al ways shrink or recede with increased conver gence. It may approach, but if it doesperceived size will decrease faster than perceived distance.

2. The expected divergence of the two functions depends not only on the magnitudes of the two types of micropsia, but also on the functions which relate perceived size and depth with visual angle and disparity respectively. Evidence from depth-size matching with variable visual angle (Gogel, 1958), however, indicates that these functions are sufficiently similar to justify interpreting the slightly smaller proportion increase in the depth-size task as indicative of a small size-micropsia effect.

3. Size micropsia presumably would influence the depth-distance settings by decreasing the perceived visual angle between the lights and thus increasing the depth-distance ratio for a given disparity. This effect, however, is sufficiently small to be considered negligible.

4. This research was supported by U.S. Public Health Service Grant MH 08878 from the National Institute of Mental Health. The author is grateful to Walter Thode for assistance in conducting these experiments and to Dr. Walter Gogel for his reading of and comments on the manuscript.

(Accepted for publication August 16, 1967.) 